\title{
INFERIOR TURBINECTOMY FOR NASAL OBSTRUCTION-STUDY OF 219 CASES
}

Santhosh U.P, Manjunatha Rao S. V.

1. Professor. Department of ENT, JJMMC, Davangere.

2. Assistant Professor. Department of ENT, Basaweshwara Medical College and Research Centre, Chitradurga, JJMMC, Davangere.

\section{CORRESPONDING AUTHOR}

Dr. Manjunatha Rao S. V,

Asst Professor, Department of ENT,

Head and Neck Surgery,

Basaweshwara Medical College and Research centre,

NH-4, Chitradurga.

E-mail: mrsarvade@yahoo.co.in

Ph: 00919620360677

ABSTRACT: OBJECTIVE: To determine the success rate of inferior turbinectomy for nasal obstruction. To study complications of inferior turbinectomy. To study the histopathological changes in the inferior turbinate specimens. METHOD: This prospective, single blinded, randomized study of 219 consecutive patients carried out in two tertiary level centres from January 2010 to October 2011. The patients were assessed preoperatively and postoperatively both subjectively and objectively. The patients who were refractory to the directed conservative treatment for chronic nasal obstruction underwent inferior turbinectomy on both sides and septoplasty if required. The inferior turbinectomy specimen was sent for histopathological examination. NOSE Questionnaires were used for subjective assessment and rigid nasal endoscopy used for objective assessment. Follow up done at 1 week, 1 month and 6 months after surgery. Friedman test applied for assessment of the improvement. RESULTS: $92 \%$ $(n=203)$ of patients relieved of nasal obstruction for which they approached. The improvement is statistically significant both subjectively and objectively. The common complications in present study is postoperative nasal synechia which occurred in $5 \%(n=10)$. Bleeding occurred in $4 \%(n=8)$. Dryness and crusting occurred in $2 \%(n=4)$ and foul odour in $2 \%(n=4)$. The present study also showed that most common cause for turbinate enlargement is chronic nonspecific inflammatory hypertrophy followed by allergic turbinate hypertrophy. CONCLUSION: -The results show that inferior turbinectomy is the good method of achieving relief from the nasal obstruction with minimal morbidity. Histopathological examination showed that most common cause of turbinate enlargement is chronic non specific turbinate hypertrophy and it proves beyond doubt that unnecessary excessive antiallergic treatment should be avoided in patients with chronic nasal obstruction.

KEY WORDS: inferior turbinectomy, chronic nonspecific turbinate hypertrophy.

INTRODUCTION: Nasal obstruction is one of the common complaint for which the patient approaches to the ENT surgeon(2,3,7). Chronic nasal obstruction has many adverse sequelae including mouth breathing, dryness of the oropharynx, nasal speech, disordered sleep, restlessness, malaise, an adverse effect on quality of life and reduced lung volumes (2,5). Nasal obstruction due to the turbinate enlargement is usually amenable to medical treatment of the 
underlying inflammatory process (2). However long standing swelling may become irreversible. This may be due to dilated submucosal venous sinuses becoming varicose and unresponsive to sympathetic nervous system stimulation or medical treatment or because of fibrosis (2). In these instances inferior turbinate surgery may be offered $(1,2)$. Turbinate surgery is common and has been reported as the eighth most common procedure performed by otolaryngologists (2). Techniques of turbinate reduction include turbinectomy, submucous turbinectomy, inferior turbinoplasty, cryotherapy, submucous electrosurgery, $\mathrm{CO}_{2}$ laser turbinoplasty and others. No technique is perfect, and each is associated with known short-term and long-term complications such as bleeding and atrophic rhinitis. The variety of surgical techniques available indicates the lack of consensus on the optimal technique(6).

PATHOPHYSIOLOGY OF TURBINATE ENLARGEMENT: The physics established with Poiseuilles law demonstrates that as little as a $10 \%$ increase in the cross sectional area of the nasal passage produces a $21 \%$ increase in flow ${ }^{(2)}$. Decongestion of the nose increases the volume of the nasal cavity by $35 \%$ (2). Turbinectomy is a conventional technique for reducing their size to achieve patent nasal airways in situations where an enlarged turbinate contributes to airway obstruction (2,8). The inferior turbinate reduction with its success and complication rates along with histopathological causes for its enlargement is described.

OBJECTIVE: The main objectives of this study was

To determine the success rate of inferior turbinectomy for nasal obstruction.

To study complications of inferior turbinectomy.

To study the histopathological changes in the inferior turbinate specimens.

MATERIAL AND METHODS: This is a prospective, single blinded, randomized study of 219 consecutive patients from January 2010 to October 2011 carried out in two tertiary level centres. These patients came with main complaint of the nasal obstruction. A detailed history taken. These patients were given course of antibiotics and nasal decongestants for 1 week. If there is no improvement after directed medical treatment, then X Ray paranasal sinuses taken to rule out sinus pathology. Patients with normal para nasal sinuses with chronic nasal obstruction were included in the study. Participants were immunocompetent, generally healthy individuals. Given that allergy tests were not carried out in all cases, no differentiation was made between patients with or without allergy.

INCLUSION CRITERIA: The patients with the history of

1. Chronic nasal obstruction due in part to inferior turbinate hypertrophy with or without nasal septal deformity.

2. Failure of medical treatment of rhinitis medicamentosa.

Inferior turbinate's graded from I to III. Grade I was defined as mild enlargement with no obvious obstruction. Grade III was complete occlusion of the nasal cavity. The turbinate's in between were graded as II. Grade II and grade III turbinate's were included in the study.

\section{EXCLUSION CRITERIA:}

1. Patients with acute rhinitis.

2. Patients with sinus pathology. 
3. Patients who responded to nasal decongestants.

4. Patients with history of crusting in the nose.

5. Patients with dryness in nose.

SURGICAL TECHNIQUE: All patients signed an informed consent form after being informed of the known benefits, risks, complications, and alternatives to surgery. All patients underwent turbinectomy of hypertrophied inferior turbinate under general anesthesia with or without septoplasty. The inferior turbinate's were injected with $8 \mathrm{ml}$ of the $2 \%$ lidocaine within $1 / 100,000$ epinephrine (Astra Zeneca) solution in a submucosal plane. Inferior turbinate medialized with freers elevator. The straight artery forceps applied and clamped and crushed. The straight artery removed and the inferior turbinate cut with inferior turbinectomy scissors. The central strategy of turbinate surgical procedures is to reduce the volume of the inferior turbinate particularly in its anterior portion, a component of the internal nasal valve, the most resistive segment of the upper airway. The nasal airflow is to be improved without affecting nasal physiology. Turbinectomy included anterior $2 / 3 \mathrm{rd}$ of turbinate and all the layers including the bone were excised during the procedure. Specimen sent for histopathological examination. Hemostasis was achieved under direct vision with suction electrocautery when necessary. Nasal packing was used for 48 hours. Complications of synechia, bleeding, crusting, foul odor and nasolacrimal duct injury were recorded.

TISSUE PREPARATION: All samples were separately collected, underwent standard processing, and were investigated microscopically. Each sample had a well-defined conchal form with clear inferior, medial, and lateral portions of soft tissue and bony structure. The samples were fixed in buffered formaldehyde, thereafter decalcified with $0.7 \mathrm{M}$ ethylenediamine tetraacetic acid (EDTA) and dehydrated with increasing concentrations of ethanol. The sections were embedded in paraffin blocks and cut. Section was stained with hematoxylin-eosin and mounted on a glass slide and studied.

Patients were followed up 1week, 1 month and at 6 month post operatively. Minimum follow-up was 6 months, with a range of 6 to 12 months. The subjective assessement and objectively both preoperatively and postoperatively using NOSE questionnaire and rigid nasal endoscopy. Friedman test applied for assessment of the improvement.

RESULTS: A total of 219 cases (148 male and 71 female patients) between the ages of 19 and 60 years with symptom of nasal obstruction were operated.

In present study 203 patients were relieved of nasal obstruction at the end of 6 months which is statistically significant both subjectively and objectively. 16 patients were not relieved of nasal blockage subjectively. Endoscopic examination in follow up showed that 4 of these patients had enlarged middle turbinate's and in others cause was not found out.

In present study 10 patients developed synechia following surgery. 8 patients had post operative bleeding. One case bleeding occurred after emergence from anesthesia. A branch of the sphenopalatine artery at the level of the choana was identified and bleeding was controlled with suction electrocautery. 5 cases had bleeding soon after removal of pack. These patients were repacked and removed it later after 2 days and bleeding controlled. One patient had bleeding 4 days later, 2days after removing pack. He had 5 pints of beer in post operative period. In this patient repacking did not stop the bleeding. It stopped only after whole blood transfusion. None of the patients had crusting or dryness in the nose before surgery, but 4 developed these symptoms after surgery. None of the patients had crusting or dryness in the 
nose before surgery, and but 4 developed these symptoms after surgery. 4 patients developed foul smell in the nose 2 months later surgery. In the present study there was no nasolacrimal duct injury complication.

In the present showed that chronic non specific turbinate hypertrophy is most common cause for turbinate hypertrophy $(\mathrm{n}=203)$ followed by chronic allergic turbinate hypertrophy $(n=5)$. A case of adenoid cystic carcinoma and lepromatous leprosy were also found.

DISCUSSION: Prolonged perceived nasal obstruction resulting from inferior turbinate ITH) is a common complaint encountered in the practice of rhinology. Several causes may induce significanthypertrophic mucosal changes of the inferior turbinate (IT), including perennial allergic rhinitis and nonallergic (vasomotor) rhinitis. The patients are usually offered conservative therapy with antihistamines, systemic decongestants, topical nasal steroid sprays, and mast cell stabilizers. When these means did not provide adequate relief for the patient, surgery was suggested(1,4). Clearly, the decision for surgery should be based not only on the clinical presentation but also on the histopathologic features of the organ. However, data on the latter are less(4). The present study provides information on effectiveness of inferior turbinate and to have qualitative analysis of the hypertrophic inferior turbinate.

In present study 203 patients were relieved of nasal obstruction. The patients had significant symptomatic relief after turbinectomy, and the present findings are consistent with other studies reported in the literature. However, concomitant nasal septal surgery with the inferior turbinate surgery in some cases limits the ability of present study to attribute the relief of nasal obstruction merely to the reduction in the size of the inferior turbinate's. 16 patients were not relieved of nasal obstruction subjectively. This may be due to damaged, resected or bypassed trigeminal nerve endings which can create the sensation of nasal obstruction despite no objective increase of nasal airway resistance. It is possible patients may have atrophy of the nerve fibres in the nasal lining subserving the sensation of nasal airflow(2). This shows that turbinate resection should be performed as conservatively as possible, but willing to do what is necessary to achieve the desired result.

In present study 10 patients developed synechia following surgery. Mucosal tears persisted as synechia to the septum. Delicate septal surgery without any mucosal damage also prevents this complication. The incidence of tears decreased significantly during the course of this study owing to the learning curve of a procedure. All the synechia were released endoscopically and gel foam kept. The bleeding can be prevented by preservation of some of the bony turbinate especially posteriorly prevents bleeding. The 4 patients developed dryness and crusting after surgery. This was managed conservatively with saline nasal douche. Foul odor developed in 4 patients is a consequence of scabbing and necrosis that is often seen during the postoperative course of inferior turbinectomy due to excessive drying from loss of turbinate mucosa, destruction of cilia secondary to scarring, atrophy and end stage infection. These patients were managed it with saline douche and glucose with glycerin nasal drops. None of the patients had nasolacrimal duct injury in present study probably due to proper illumination during surgery and meticulous handling.

Understanding the histology of the hypertrophic inferior turbinate is imperative for the development and management of inferior turbinate reduction surgery. The normal turbinate consists of an outer layer of respiratory mucosa, a submucosal layer, an inner periosteal layer and the turbinate bone. The submucosal layer is largely venous sinusoids, capable of engorgement with blood, causing swelling of the nasal mucosa $(3,5)$. The present showed that 
chronic non specific turbinate hypertrophy is most common cause for turbinate hypertrophy $(n=203)$ followed by chronic allergic turbinate hypertrophy $(n=5)$. This raises question regarding excessive use of treatment directed against allergy. A case of adenoid cystic carcinoma and lepromatous leprosy were also found. This shows that every specimen we remove from the nose should be sent for histopathological examination

CONCLUSION: The study demonstrates that inferior turbinectomy effective surgery in reliving the nasal obstruction. The complications are common but are minimal and can be effectively managed conservatively. However, the relative short follow-up is insufficient to make definitive statements about the long-term outcome. With results of histopathological analysis it is concluded that chronic non specific turbinate hypertrophy is the most common cause of turbinate hypertrophy and unnecessary excessive antiallergic treatment should be avoided in patients with chronic nasal obstruction. Sending the inferior turbinate specimen for histopathological examination is justified seeing the rare diagnosis found in the study. Future well designed studies involving prospective data collection, validated outcome measures, and long-term follow-up are required.

\section{BIBILOGRAPHY:}

1. Batra PS, Seiden AM, Smith TL(2009). Surgical management of adult inferior turbinate hypertrophy: a systematic review of the evidence. Laryngoscope 119:1819-1827.

2. David Willatt(2009). The evidence for reducing inferior turbinate's. Rhinology, 47, 227236.

3. Farmer, Eccles(2006). Chronic inferior turbinate enlargement and the implications for surgical intervention ${ }_{\llcorner}$Rhinology, 44, 234-238.

4. Gilead Berger (2006) The histopatholgy of the hypertrophic inferior turbinate Arch Otolaryngol Head Neck Surg. 132:588-594

5. John Mathai(2004) Inferior turbinectomy for nasal obstruction review of 75 cases. Indian journal of otolaryngology and head and neck surgery. 56.1. 23-26.

6. Michael Friedman, Hasan Tanyeri, Jessica Lim, Roy Landsberg, David Caldarelli,(1999) A Safe, Alternative Technique for Inferior Turbinate Reduction Laryngoscope_109: November 18-34.

7. Ottaviani F, Capaccio P,Cesana BM, et al.(2003) Argon plasma coagulation in the treatment of non allergic hypertrophic inferior nasal turbinate's. American Journal of Otolaryngol. 24:306-310.

8. Ozcan KM, Gedikli Y, Ozcan I, et al(2008). Microdebrider for reduction of inferior turbinate: evaluation of effectiveness by computed tomography. Journal of Otolaryngol Head Neck Surg. 2008; 37:463-468. 
Table I summarizes the subjective data gathered from the questionnaires and comparison with various authors

\begin{tabular}{|l|l|l|l|l|l|l|}
\hline & $\begin{array}{l}\text { Martinez et } \\
\text { al 1983 }\end{array}$ & $\begin{array}{l}\text { Moore et } \\
\text { al 1985 }\end{array}$ & $\begin{array}{l}\text { Percadoni } \\
\text { etal 1996 }\end{array}$ & $\begin{array}{l}\text { Rakover } \\
\text { etal 1996 }\end{array}$ & $\begin{array}{l}\text { John } \\
2004\end{array}$ & $\begin{array}{l}\text { Present } \\
\text { series }\end{array}$ \\
\hline $\begin{array}{l}\text { Nasal block } \\
\text { improvement }\end{array}$ & $100 \%$ & $27 \%$ & $90 \%$ & $77 \%$ & $98 \%$ & $92 \%$ \\
\hline Synechia & & & & $2.6 \%$ & $3 \%$ & $5 \%$ \\
\hline Bleeding and & $13 \%$ & $89 \%$ & - & $2.6 \%$ & $3 \%$ & $4 \%$ \\
\hline $\begin{array}{l}\text { Dryness } \\
\text { crusting }\end{array}$ & 0 & $39 \%$ & - & - & $8 \%$ & $2 \%$ \\
\hline Foul smell & 0 & - & - & - & 0 & $2 \%$ \\
\hline $\begin{array}{l}\text { Nasolacrimal } \\
\text { duct injury }\end{array}$ & - & & & & 0 & 0 \\
\hline
\end{tabular}

TABLE II showing histopathological diagnosis and no of the patients

\begin{tabular}{|l|l|}
\hline Histopathological diagnosis & No of patients \\
\hline Chronic non specific turbinate hypertrophy & 203 \\
\hline Allergic turbinate hypertrophy & 5 \\
\hline Chronic inflammatory polyp & 2 \\
\hline Allergic polyp & 1 \\
\hline Rhinoscleroma & 1 \\
\hline Adenoid cystic carcinoma & 1 \\
\hline Angioma & 1 \\
\hline Pyogenic granuloma & 1 \\
\hline Myofibroblastic sarcoma & 1 \\
\hline Lepromatous leprosy & 1 \\
\hline Rhinolith & 1 \\
\hline Capillary hemangioma & 1 \\
\hline
\end{tabular}

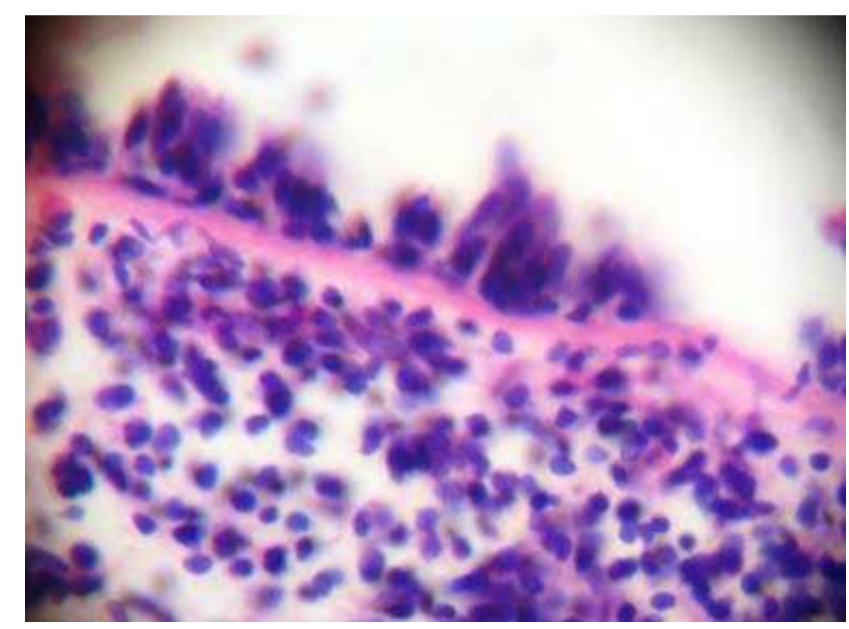

Chronic non specific turbinate hypertrophy 